25th SOFT, 15 - 19 September 2008, Rostock, Id. Nr. P-279

First author: Wolfgang Treutterer, Boltzmannstraße 2, D-85748 Garching, Germany

email: Treutterer@ipp.mpg.de; phone: ++49/89/3299-1496, fax:++49/89/3299-2580

\title{
Real-Time Diagnostic Integration with the ASDEX Upgrade Control System
}

W. Treutterer, L. Giannone, K. Lüddecke*, G. Neu, G. Raupp, D. Zasche, T. Zehetbauer and ASDEX Upgrade Team

Max-Planck-Institut für Plasmaphysik, EURATOM Association, Boltzmannstraße 2, D-85748

Garching, Germany

*Unlimited Computers GmbH, Seeshaupterstraße 15, D-82393 Iffeldorf, Germany

\begin{abstract}
Operating in advanced plasma scenarios has become one of the major goals on the way towards ITER and a future fusion reactor. This implies the reinforced control of physics quantities like pressure profiles or magnetic island structures, which have to be reconstructed in real-time for this purpose. To achieve this goal the collaboration scheme between diagnostic data processing and real-time control has to be fundamentally changed from strict separation to open bidirectional information exchange in real-time.

ASDEX Upgrade is currently designing and building a distributed computer cluster to implement such an integrated diagnostic and control system. The main topics comprise modular framework design, low-latency data communication via networks, and built-in synchronization methods.

We show how the discharge control system's modular framework is extended to publish calculated quantities via the network transparent to algorithm designers. A communication layer allows the exchange of real-time information between computation nodes with automatised routing even over several networks. Finally, in order to consistently combine data from independent sources, synchronization methods are developed. Thus, sophisticated feedback control with time scales of milliseconds become feasible.
\end{abstract}




\section{Introduction}

While real-time evaluation and control of plasma current, position and shape as well as averaged electron density are classical components of a tokamak experiment's control system, many other quantities are typically reconstructed only after a discharge from collected raw sensor data. Control system and diagnostic plant systems were disjunct. Inputs required by the control system were basically replicas of analog sensor signals. This separation allowed for independent and robust operation of control and diagnostic systems.

Due to limited information about the plasma state, however, the control system was not able to advance into and stabilise high performance scenarios or detect critical situations in time. The growing knowledge about plasma core and edge physics must be transferred to real-time control algorithms to achieve these goals. And ITER will rely on such advanced control methods even for its standard operation.

A typical example of advanced control and monitoring is plasma current profile control to establish transport barriers and boost confinement. Others applications serve for device protection like hot-spot detection by video monitoring, radiation peaking detection from Bolometry, or neoclassical tearing mode (NTM) control with electron cyclotron current drive (ECCD) [1], a project that is a major driving force in ASDEX Upgrade's real-time diagnostic integration efforts [2] [3].

All these examples have in common, that the relevant physics quantities to be controlled or monitored require raw measurements with a high number of sensors at high sampling rates. The classical separation of control and diagnostic systems would duplicate signal digitising and distribution hardware and the need for consistently maintaining two variants of evaluation algorithms, one for off-line diagnostic purposes and another for real-time control - a prohibitive expense. Instead, modern data acquisition and networking techniques, alongside with the need to save investment, suggest to shift paradigms towards an integrated concept of real-time data acquisition, evaluation and, finally, feedback control. 
This integration also entails risks for the reliability of the control system and hence the plant. The complexity of tasks and algorithms as well as the number of involved systems grows rapidly and with it the susceptibility to failure. The component technologies will become much more heterogeneous as diagnostic systems often require dedicated hardware platforms, come with their own operating systems and connection technology. The integration concept must account for this variety but respect the limited development resources. Hard- and software standards can alleviate the problem but should not preclude innovative solutions.

Besides providing connectivity between the participating systems, the problem of synchronising data samples has to be addressed to ensure that algorithms with mixed input from several sources can compute consistent data sets. This is particularly necessary, as the sampling periods of diagnostic systems vary considerably.

Based on the example of NTM mode control we will subsequently describe the approach ASDEX Upgrade has taken to realise this integration.

\section{Overview on NTM mode control}

NTM mode control has the aim to suppress or at least mitigate a neoclassical tearing mode which otherwise would reduce the plasma pressure or could even lock and trigger a disruption. The principle consists in direct current drive or in heating the plasma to increase local conductivity at the location of the magnetic island connected to the NTM [1]. To accomplish this, movable mirrors under the command of the NTM controller guide electron cyclotron beams to be absorbed on the magnetic flux surface where the island is centered. The controller needs information about the locations of the mode, $\rho_{\mathrm{NTM}}$, and the gyrotron power deposition, $\rho_{\mathrm{ECH}}$. These quantities can be derived in several ways by combining diagnostic measurements. A Bayesian filter is employed to compute trusted values from the available variants. The basic information flow is shown in figure 1, where the blocks connected to data acquisition hardware represent the participating diagnostic systems. Without going into detail one can immediately see, how the data of many systems have to be convoluted and end up in the commands for 
ECRH gyrotron powers and mirror positions. Raw-data sampling rates vary from $2 \mathrm{MHz}$ (ECE) over $10 \mathrm{kHz}$ (magnetic diagnostic MAX) to $1 \mathrm{kHz}$ (discharge control system DCS). These raw measurements, however, must be condensed by correlation, filtering and averaging to eliminate noise. At the end a $10 \mathrm{~ms}$ control cycle is desired for NTM control. For other purposes a shorter refresh time might be required, though.

\section{Extension of the DCS control framework}

Figure 1 shows that the same information is often used by several applications. E.g. the magnetic flux matrix $\Psi_{\text {pol }}(\mathrm{r}, \mathrm{z})$ and their derivatives are base for many transformation algorithms. Moreover, with the evolution and refinement of algorithms clients come and leave. The core of the integration concept therefore extends some of the basic ideas of ASDEX Upgrade's discharge control system DCS [4] towards real-time diagnostic systems: timestamped and qualified signal samples, global availability of plant states, and separation of application algorithms and framework services like automatic data routing and transport. The modular framework makes communication of calculated quantities via the network transparent to algorithm designers. Applications can be formulated in a self-contained manner, their only interface points being their input and output signals, without explicit knowledge of producers or consumers. Also real-time diagnostics or real-time diagnostic data processors are considered to be instances of such applications.

This encapsulation has several advantages. Applications without attached peripheral hardware can be freely placed on the most adequate computation node. Changes in the composition of signals do not affect other applications. Finally, the synchronisation problem can be solved in an elegant way. Since all signal sample requests are passed through the framework interface, the framework library can interpolate sample values to the desired point in time.

Figure 2 shows the NTM control example in a component oriented view. Real-time diagnostic systems and data processors provide platforms to execute evaluation algorithms. The results are distributed via the real-time diagnostic data network, which in fact comprises several networks 
and several technologies like Ethernet or reflective shared memory. A diagnostic server, an extension of the DCS framework, supplies a local cache with history for signal sample data from both, control and diagnostic systems. It hosts special framework "gate"-processes connecting each network to this cache (figure 3). The diagnostic server allows to combine different network technologies. It keeps intermediate results from diagnostic systems localised in its cache and thus allows to reduce data traffic on the discharge control system (DCS) realtime network.

\section{Real-time diagnostic interface}

In contrast to genuine discharge control applications, real-time diagnostic applications are developed and maintained as separate packages outside the control system code base. Nevertheless, for flawless interaction they must adhere to common operation standards. One of them is the publish-subscribe phase: prior to a discharge each application must send information about its input and output signals to a central instance which in turn calculates the signal routing and sets up the framework's communication layer accordingly. Other standards define static parameter exchange and validation, error reporting, and data archiving. In the classical integration approach a software library implements the interface to the framework, hiding all internal complexity and shielding the partners from trivial faults of the other side. Such an integration library is available for so-called standard real-time diagnostic systems. These use a standardised self-description called shotfile header [5] and run on a Solaris operating system (figure 4). A real-time diagnostic control process (rtDiagCtrl) handles all communication with the gate process on the server and other framework instances like the publish-subscribe server, the parameter server, and the logging server. The actual diagnostic algorithm is operated as a parallel process. Both processes communicate via a shared memory region where control flow commands, global parameters and signal samples are exchanged. A library of about 10 functions, the rtDiagLib, hides this mechanism from the actual diagnostic 
algorithm, which can be coded in Fortran, $\mathrm{C}$ or $\mathrm{C}++$ and, optionally, parallelised on a computer cluster with the message passing interface (MPI) framework.

For other, innovative but non-standard diagnostic systems, like the high performance LabVIEW RT®-based computers employed by the magnetic diagnostic MAX [6] a second integration path has been chosen: the minimal standards of interaction will be defined, and it will the responsibility of the diagnostic system to implement them properly. Thus the framework is open for alternative systems, but the expertise for integration can be delegated to experts for the respective systems.

\section{Summary and outlook}

ASDEX Upgrade has committed itself to investigate advanced plasma operation scenarios in preparation for ITER. This requires to use diagnostic information in real-time control on a large scale. We have discussed the requirements and developed a concept for reliable and extensible integration of heterogeneous real-time diagnostic systems with the discharge control system. Extensions of the DCS framework have been developed to connect diagnostic acquisition systems as well as diagnostic data processors with the control system's shared signal sample pool.

NTM mode control is ASDEX Upgrade's pilot project implementing this concept. Both standard diagnostic systems with full framework support and alternative LabVIEW RT based systems are involved. Basic components of the real-time data network and the framework extensions for this project are nearly complete. Tests and commissioning are planned for December 2008.

\section{References}

[1] A. Manini, J. Berrino, S. Cirant, G. D'Antona, F. Gandini, G. Grünwald, F. Leuterer, M. Maraschek, F. Monaco, G. Neu, G. Raupp, D. Sormani, J. Stober, W. Suttrop, W. Treutterer, D. Wagner, H. Zohm, ASDEX Upgrade Team, 
Development of a Feedback System to Control MHD Instabilities in ASDEX Upgrade, Fusion Engineering and Design 82 (2007), pp. 995-1001

[2] W. Treutterer, K. Behler, L. Giannone, N. Hicks, A. Manini, M. Maraschek, G. Raupp, M. Reich, A. C. C. Sips, J. Stober, W. Suttrop, ASDEX Upgrade Team, Real-time Diagnostics at ASDEX Upgrade - Integration with MHD Feedback Control, Fusion Engineering and Design 83(2-3) (2008), pp. 300-303

[3] K. Behler, H. Blank, H. Eixenberger, H. Lohs, K. Lüddecke, R. Merkel, G. Raupp, G. Schramm, W. Treutterer, M. Zilker, ASDEX Upgrade Team, Real-time Diagnostics at ASDEX Upgrade - Architecture and Operation, Fusion Engineering and Design 83(2-3) ( 2008), pp. 304-311

[4] G. Raupp, K. Behler, H. Blank, A. Buhler, R. Drube, H. Eixenberger, K. Engelhardt, A. Lohs, R. Merkel, G. Neu, G. Schramm, W. Treutterer, D. Zasche, T. Zehetbauer, ASDEX Upgrade CODAC Overview, this conference

[5] K. Behler, H. Blank, A. Buhler, R. Drube, H. Friedrich, K. Förster, K. Hallatschek, P. Heimann, F. Hertweck, J. Maier, R. Merkel, M.G. Paco-Düchs, G. Raupp, H. Reuter, U. Schneider-Maxon, R. Tisma, M. Zilker, Computer Science Division, Garching Computer Centre, ASDEX Upgrade Team, Review of the ASDEX Upgrade data acquisition environment - present operation and future requirements, Fusion Engineering and Design 43 (1999), pp. 247-258

[6] L. Giannone, W. Schneider, P. J. McCarthy, A. C. C. Sips, W. Treutterer, K. Behler, T. Eich, J. C. Fuchs, N. Hicks, A. Kallenbach, M. Maraschek, A. Mlynek, G. Neu, G. Pautasso, G. Raupp, M. Reich, K. H. Schuhbeck, J. Stober, F. Volpe, T. Zehetbauer and ASDEX Upgrade Team, Real time magnetic probe and flux loop measurements for tokamak control with a multi-core PCIe system, this conference 


\section{Figure Captions}

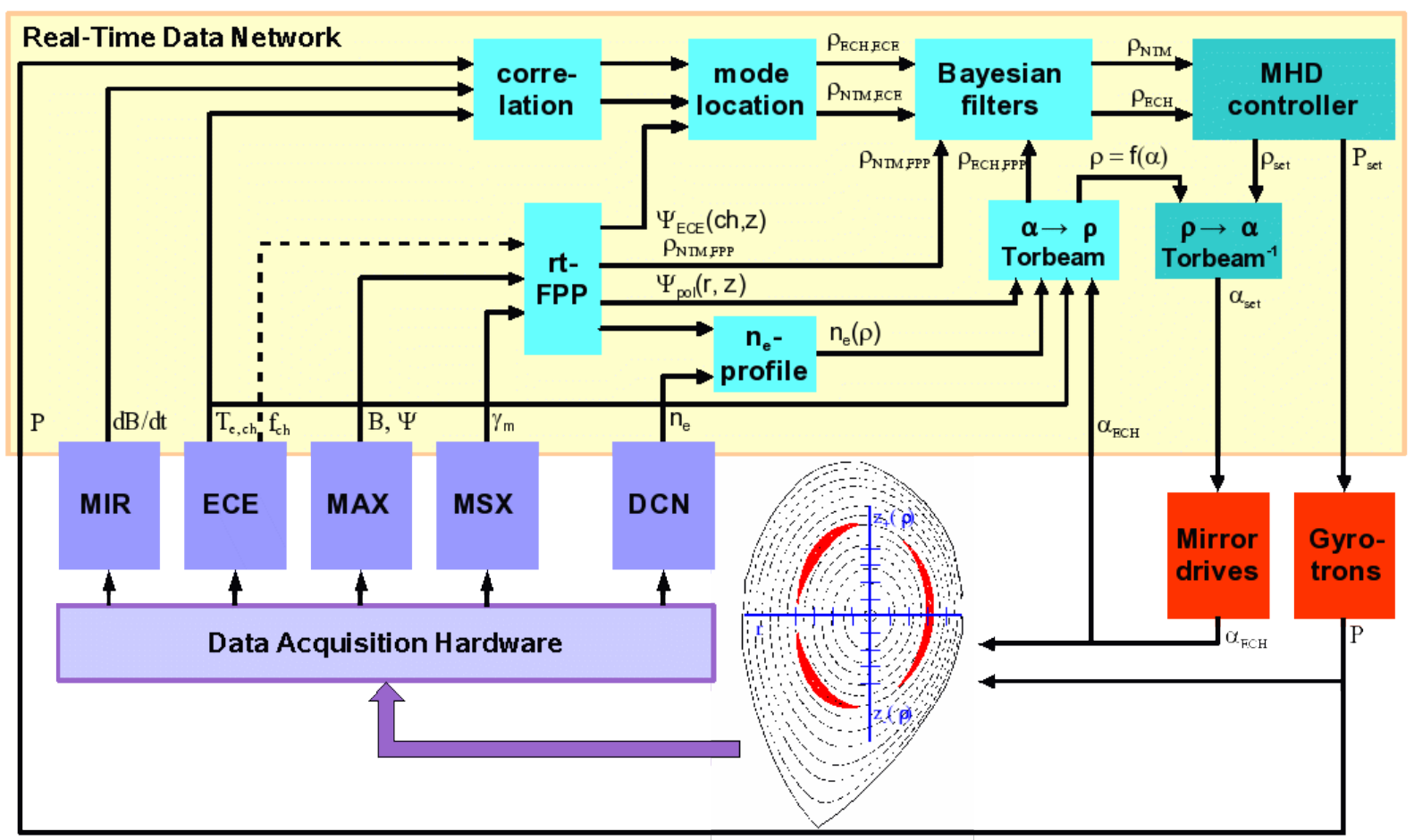

Figure 1 : NTM control overview scheme

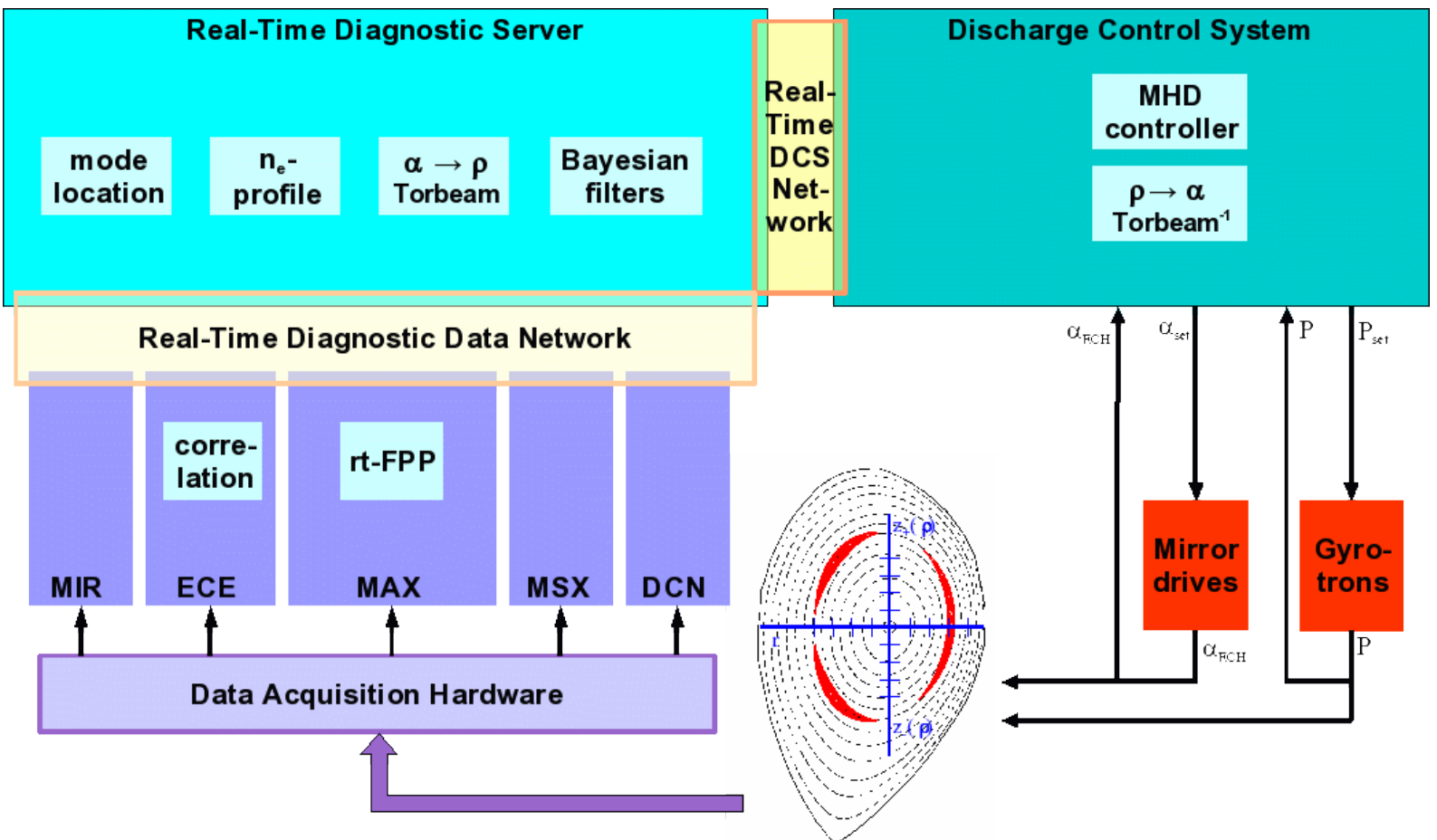

Figure 2 : NTM control component overview 


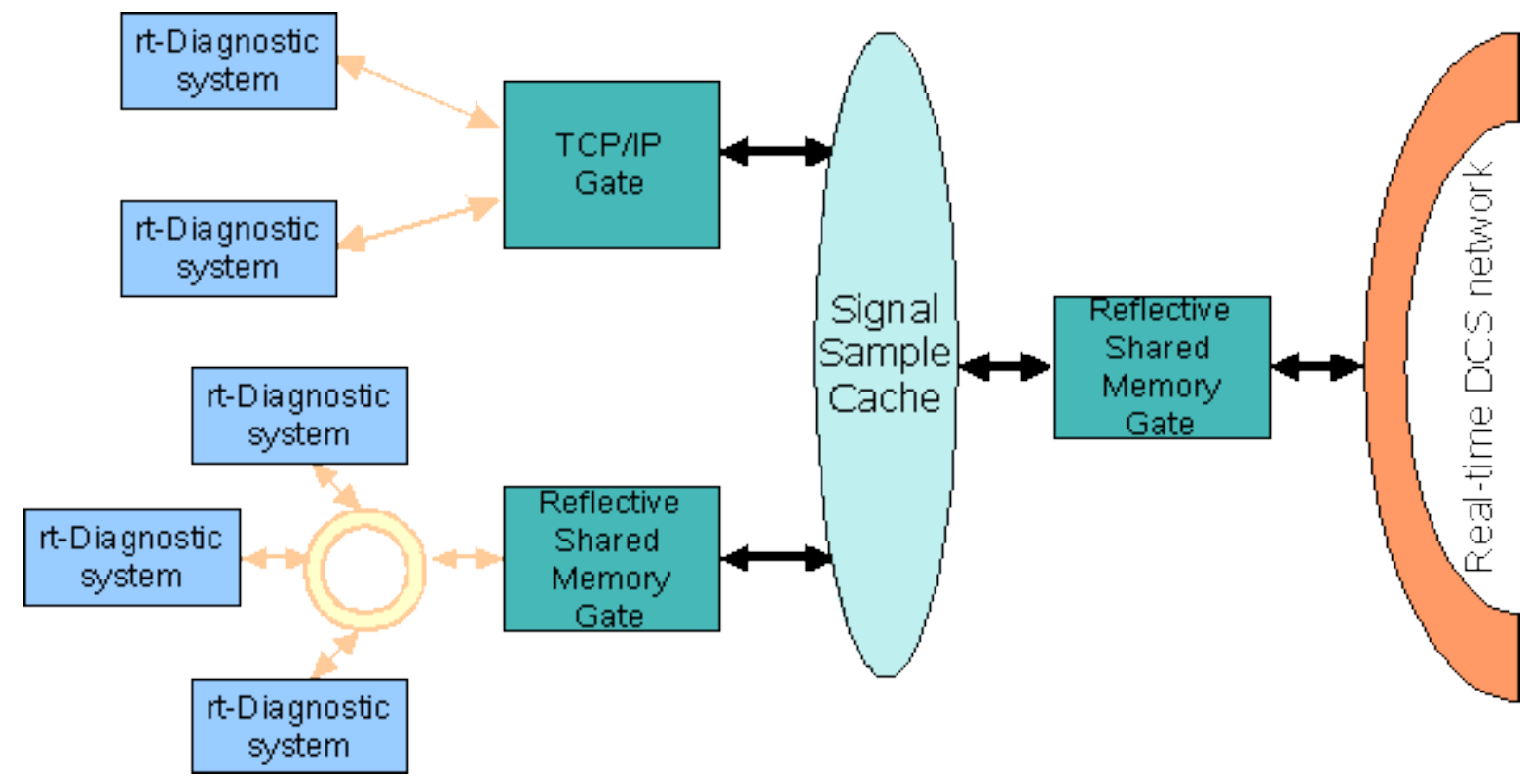

Figure 3 : Shared signal sample pool access on the diagnostic server

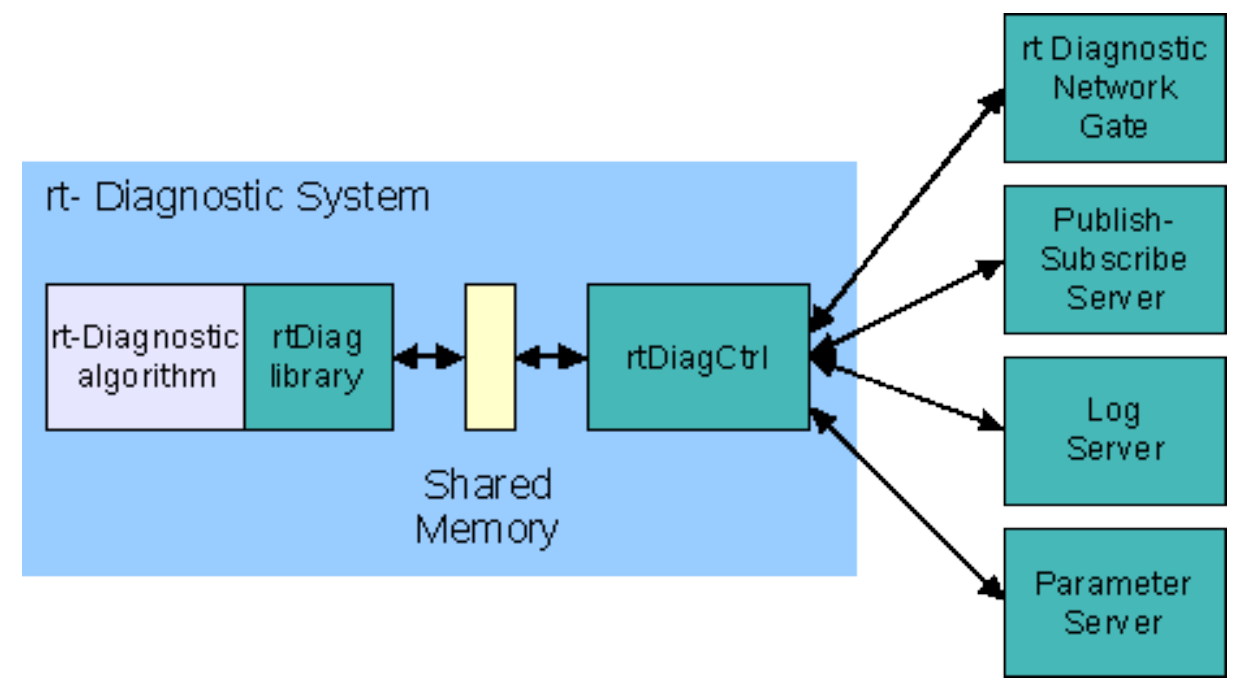

Figure 4 : Framework environment for standard real-time diagnostics 